\title{
Ferrotoroidic Moment as a Quantum Geometric Phase
}

\author{
C. D. Batista, ${ }^{1}$ G. Ortiz, ${ }^{2}$ and A. A. Aligia $^{3}$ \\ ${ }^{1}$ Theoretical Division, Los Alamos National Laboratory, P.O. Box 1663, Los Alamos, NM 87545, USA \\ ${ }^{2}$ Department of Physics, Indiana University, Bloomington, IN 47405, USA \\ ${ }^{3}$ Comisión Nacional de Energía Atómica, Centro Atómico Bariloche and Instituto Balseiro, \\ 8400 S.C. de Bariloche, Argentina
}

(Dated: October 29, 2018)

\begin{abstract}
We present a geometric characterization of the ferrotoroidic moment $\boldsymbol{\tau}$ in terms of a set of Abelian Berry phases. We also introduce a fundamental complex quantity, $z_{\mu \nu}$, which provides an alternative way to calculate $\tau$ and its moments and is derived from the tensor $T_{\mu \nu}=2 \sum_{j} r_{j}^{\mu} S_{j}^{\nu}$. This geometric framework defines a natural computational approach for density functional and many-body theories.
\end{abstract}

PACS numbers: 03.65.Vf, 75.80.+q, 03.65.-w

Introduction. The recent discovery of new compounds with strong multiferroic coupling revised interest in multiferroic materials. An attractive component of this discovery is the potential for realizing strong magnetoelectric effects, i.e., magnetic field (B) induced electric polarization or electric field (E) induced magnetic moment. The linear magnetoelectric response is characterized by the magnetoelectric tensor $\hat{\alpha}[1]$, and different physical mechanisms can contribute to it. One of these mechanisms has been recently the focus of considerable attention [2, 3, 4, 5, 6, 7] and is related to the concept of toroidic moment [8]. Materials with a uniform toroidic moment (ferrotoroidics) exhibit a non-vanishing magnetoelectric effect, and the ferrotorodic moment is an antisymmetric component of $\hat{\alpha}$. These materials might have technological applications in the area of computer memories.

The observation of ferrotoroidic domains in $\mathrm{LiCoPO}_{4}$ [6] confirmed the physical relevance of this concept and opened the possibility of observing toroidic driven magnetoelectric effects in certain quantum magnets. However, as it was pointed out recently [], the toroidic moment is multivalued in a periodic system. This problem is analogous to the case of the electric polarization. The root of this indeterminacy lies in the multivalued nature of the position operator for a periodic system: $\mathbf{r} \equiv \mathbf{r}+\boldsymbol{a}$, where $\boldsymbol{a}$ is a translation that leaves the system invariant [9]. The position operator appears explicitly in the expressions for the multipolar moments of charge or current distributions and, thus, all of these moments are multivalued in a periodic system. In particular, the toroidic moment appears in a multipolar expansion of a current density distribution and it couples linearly to $\nabla \times \mathbf{B}[8]$, and to $\mathbf{E} \times \mathbf{B}$ [10], which is easier to control.

Since it is usually convenient to use periodic boundary conditions for modeling physical systems, it is necessary to know how to compute the ferrotoroidic moment, $\tau$, and the consequent magnetoelectric effect within this framework. Although $\tau$ is multivalued for a periodic system, the change of $\boldsymbol{\tau}(\boldsymbol{\Delta} \boldsymbol{\tau})$ between two different physical states is well defined when moving along a particular path. In the case of the macroscopic electric polarization, $\mathbf{P}$, of an insulator, the change $\Delta \mathbf{P}$ is computed by integrating the charge current through a given surface along an adiabatic path that connects the two different physical states [9, 11]. This leads to a natural relation between $\Delta \mathbf{P}$ and the Berry phase associated to an adiabatic evolution in an enlarged parameter space [11, 12], and illustrates the observable character of the geometric phase. In the same way that the charge Berry phase $\gamma^{c}$ is a measure of the macroscopic electric polarization in band or Mott insulators [11], the spin Berry phase $\gamma^{s}$ can be related to the difference between electrical polarizations for spin up and down [13]. Here we will demonstrate that the change in the ferrotoroidic moment, $\Delta \boldsymbol{\tau}$, can be related to a Berry phase tensor $\gamma_{\mu \nu}^{s}$, thus revealing the geometric character of the ferrotoroidic response.

Ferrotoroidic moment. Assume a system of $N$ interacting electrons of mass $m$ and charge $e$ enclosed in a $d$-dimensional box of linear dimension $L_{\mu}$ and volume $\Omega(\mu=1, \cdots, d)$. Particle $j$ is labeled by the coordinates $r_{j}^{\mu}$ and spin $S_{j}^{\nu}=\frac{1}{2} \sigma^{\nu}\left(\sigma^{\nu}\right.$ are Pauli matrices with $\nu=x, y, z)$. The Hamiltonian of the system is $(\hbar=1)$

$$
\begin{aligned}
H^{\lambda} & =\sum_{i=1}^{N} \frac{\boldsymbol{\Pi}_{i}^{2}}{2 m}+\sum_{i<j} V_{\mathrm{int}}\left(\left|\mathbf{r}_{i}-\mathbf{r}_{j}\right|\right)+\sum_{i=1}^{N} v_{\mathrm{ext}}\left(\mathbf{r}_{i}, \lambda(t)\right) \\
& +g_{\mathrm{so}} \sum_{i=1}^{N} \mathbf{U}^{\dagger}\left(\phi_{\mu \nu}\right) \boldsymbol{\sigma}_{i} \mathbf{U}\left(\phi_{\mu \nu}\right) \cdot \mathbf{r}_{i} \times \boldsymbol{\Pi}_{i}
\end{aligned}
$$

where $\boldsymbol{\Pi}_{i}=\mathbf{p}_{i}+\mathbf{A}^{\nu}$ is the canonical momentum $\left(p_{\mu}=\right.$ $\left.-i \partial_{\mu}\right)$ with gauge field $\mathbf{A}^{\nu}=\frac{\phi_{\mu \nu}}{L_{\mu}} \sigma^{\nu} \hat{\mathbf{e}}_{\mu}\left(\hat{\mathbf{e}}_{\mu}\right.$ is a unit vector along the $\mu$ direction). The flux $\phi_{\mu \nu}$ is the twist in spin space at the boundary of the box: $\Psi_{\alpha}\left(\mathbf{r}_{i}+L_{\mu} \hat{\mathbf{e}}_{\mu}\right)=$ $\sum_{\beta}\left[e^{i \phi_{\mu \nu} \sigma^{\nu}}\right]_{\alpha \beta} \Psi_{\beta}\left(\mathbf{r}_{i}\right)$.

The transformation defined by $\mathbf{U}\left(\phi_{\mu \nu}\right)=$ $e^{i \phi_{\mu \nu} \sigma^{\nu} \sum_{j} r_{j}^{\mu} / L_{\mu}}$ is such that the eigenstates of $H^{\lambda}$ satisfy periodic boundary conditions. The last term in (11) corresponds to the relativistic spin-orbit interaction, while $V_{\text {int }}$ and $v_{\text {ext }}$ represent the interaction and "external" potentials, respectively. The latter depends parametrically on $\lambda(t)$ which is assumed to change adiabatically in time $t$ from $\lambda(0)=0$ to $\lambda(T)=1$.

The second quantized form of $H^{\lambda}$ is $\mathbb{H}^{\lambda}=$ 
$\int_{\Omega} d^{3} r \mathcal{H}^{\lambda}(\mathbf{r})$, with Hamiltonian density

$$
\begin{aligned}
\mathcal{H}^{\lambda}(\mathbf{r}) & =\boldsymbol{\Psi}^{\dagger}(\mathbf{r}) \frac{\boldsymbol{\Pi}^{2}}{2 m} \boldsymbol{\Psi}(\mathbf{r})+\mathcal{H}_{\mathrm{int}}(\mathbf{r})+\mathcal{H}_{\mathrm{ext}}^{\lambda}(\mathbf{r}) \\
& +g_{\mathrm{so}} \boldsymbol{\Psi}^{\dagger}(\mathbf{r}) \mathbf{U}^{\dagger}\left(\phi_{\mu \nu}\right) \boldsymbol{\sigma} \mathbf{U}\left(\phi_{\mu \nu}\right) \cdot \mathbf{r} \times \boldsymbol{\Pi} \boldsymbol{\Psi}(\mathbf{r}),(2)
\end{aligned}
$$

written in terms of the fermionic spinor fields $\boldsymbol{\Psi}^{\dagger}(\mathbf{r})=$ $\left(\psi_{\uparrow}^{\dagger}(\mathbf{r}), \psi_{\downarrow}^{\dagger}(\mathbf{r})\right)$, where $\psi_{\alpha}^{\dagger}(\mathbf{r})\left(\psi_{\beta}(\mathbf{r})\right)$ creates (annihilate) an electron with spin $\alpha(\beta)=\{\uparrow, \downarrow\}$ at position $\mathbf{r}$.

The spin contribution to the ferrotoroidic moment $\tau_{\nu}$ is defined as the antisymmetric component of the tensor $t_{\mu \nu}$. The $t_{\mu \nu}$-density field is defined by the local condition $\sum_{\mu} \partial_{\mu} \mathcal{T}_{\mu \nu}(\mathbf{r})=-2 \mathcal{S}_{\nu}(\mathbf{r})$. For a finite system with open boundary conditions (surfaces), the following relation holds as long as $\mathcal{T}_{\mu \nu}(\mathbf{r})$ vanishes outside the system

$$
t_{\mu \nu}=\frac{T_{\mu \nu}}{\Omega}=\frac{2}{\Omega} \int_{\Omega} d^{3} r r_{\mu} \mathcal{S}_{\nu}(\mathbf{r})=\frac{1}{\Omega} \int_{\Omega} d^{3} r \mathcal{T}_{\mu \nu}(\mathbf{r}),
$$

written in terms of the spin density field

$$
\mathcal{S}_{\nu}(\mathbf{r})=\sum_{\alpha \beta} S_{\alpha \beta}^{\nu}(\mathbf{r}), \mathcal{S}_{\alpha \beta}^{\nu}(\mathbf{r})=\frac{1}{2} \psi_{\alpha}^{\dagger}(\mathbf{r}) \sigma_{\alpha \beta}^{\nu} \psi_{\beta}(\mathbf{r}),
$$

where $\int_{\Omega} d^{3} r \mathcal{S}_{\nu}(\mathbf{r})=0$ to have $t_{\mu \nu}$ independent of the origin of coordinates [14]. More specifically $\tau_{\eta}=$ $\frac{\mu_{B}}{4} \sum_{\mu \nu} \epsilon_{\eta \mu \nu} t_{\mu \nu}$, with $\epsilon_{\eta \mu \nu}$ the Levi-Civita tensor. Like for the electric polarization [11, 15], the third member of Eq. (3) is not well defined for a system with periodic boundary conditions because the position operator is not well defined [9]. However, the last member of Eq. (3) is still well defined, and starting from such expression for $t_{\mu \nu}$, we will demonstrate that the change $\left\langle\Delta t_{\mu \nu}\right\rangle$ between two different physical states can be obtained from a set of Abelian Berry phases.

Since the ground state (GS) of $H^{\lambda}$ evolves continuously between $\lambda(0)=0$ and $\lambda(T)=1$, the change is given by

$$
\left\langle\Delta t_{\mu \nu}\right\rangle=\frac{1}{\Omega} \int_{0}^{1} d \lambda \int_{\Omega} d^{3} r \partial_{\lambda}\left\langle\Phi_{0}^{\lambda}\left|\mathcal{T}_{\mu \nu}(\mathbf{r})\right| \Phi_{0}^{\lambda}\right\rangle,
$$

where $\left|\Phi_{0}^{\lambda}\right\rangle$ is the GS of $\mathbb{H}^{\lambda}$ with flux $\phi_{\mu \nu}$, and $\left\langle\Delta t_{\mu \nu}\right\rangle=$ $\left\langle\Phi_{0}^{\lambda(T)}\left|t_{\mu \nu}\right| \Phi_{0}^{\lambda(T)}\right\rangle-\left\langle\Phi_{0}^{\lambda(0)}\left|t_{\mu \nu}\right| \Phi_{0}^{\lambda(0)}\right\rangle$. By using adiabatic perturbation theory [11, 16] up to first order in the time derivative we obtain

$$
\begin{aligned}
& \frac{1}{\Omega} \int_{\Omega} d^{3} r \partial_{\lambda}\left\langle\Phi_{0}^{\lambda}\left|\mathcal{T}_{\mu \nu}(\mathbf{r})\right| \Phi_{0}^{\lambda}\right\rangle= \\
& -\frac{i L_{\mu}}{\Omega} \sum_{m \neq 0} \frac{\left\langle\Phi_{0}^{\lambda}\left|\partial_{\phi_{\mu \nu}} H^{\lambda}\right| \Phi_{m}^{\lambda}\right\rangle\left\langle\Phi_{m}^{\lambda}\left|\partial_{\lambda} \mathbb{H}^{\lambda}\right| \Phi_{0}^{\lambda}\right\rangle}{\left[E_{0}^{\lambda}-E_{m}^{\lambda}\right]^{2}}+\text { c.c., }
\end{aligned}
$$

where we have used that $\mathbb{H}^{\lambda}\left|\Phi_{m}^{\lambda}\right\rangle=E_{m}^{\lambda}\left|\Phi_{m}^{\lambda}\right\rangle$ $\left(\left\langle\Phi_{m}^{\lambda} \mid \Phi_{m^{\prime}}^{\lambda}\right\rangle=\delta_{m, m^{\prime}}\right)$ and

$$
i\left[\mathbb{H}^{\lambda}, \mathcal{T}_{\mu \nu}(\mathbf{r})\right]=\partial_{t} \mathcal{T}_{\mu \nu}=L_{\mu} \partial_{\phi_{\mu \nu}} \mathcal{H}^{\lambda}(\mathbf{r}),
$$

In the absence of the spin-orbit term, $\partial_{t} \mathcal{T}_{\mu \nu}=2 \mathcal{J}_{\mu \nu}^{s}(\mathbf{r})$ with $\mathcal{J}_{\mu \nu}^{s}(\mathbf{r})$ the density of spin current [17] satisfying the continuity equation $\sum_{\mu} \partial_{\mu} \mathcal{J}_{\mu \nu}^{s}(\mathbf{r})+\partial_{t} \mathcal{S}_{\nu}(\mathbf{r})=0$. By using the relations $\left\langle\Phi_{m}^{\lambda}\left|\partial_{\lambda} \mathbb{H}^{\lambda}\right| \Phi_{0}^{\lambda}\right\rangle=\left\langle\Phi_{m}^{\lambda}\left|\left[\partial_{\lambda}, \mathbb{H}^{\lambda}\right]\right| \Phi_{0}^{\lambda}\right\rangle$, and $\left\langle\Phi_{0}^{\lambda}\left|\partial_{\phi_{\mu \nu}} H^{\lambda}\right| \Phi_{m}^{\lambda}\right\rangle=\left\langle\Phi_{0}^{\lambda}\left|\left[\partial_{\phi_{\mu \nu}}, H^{\lambda}\right]\right| \Phi_{m}^{\lambda}\right\rangle$, we obtain from Eqs. (5) and (6)

$$
\begin{aligned}
\left\langle\Delta t_{\mu \nu}\right\rangle & =-\frac{i L_{\mu}}{\Omega} \int_{0}^{1} d \lambda \sum_{m \neq 0}\left\langle\partial_{\phi_{\mu \nu}} \Phi_{0}^{\lambda} \mid \Phi_{m}^{\lambda}\right\rangle\left\langle\Phi_{m}^{\lambda} \mid \partial_{\lambda} \Phi_{0}^{\lambda}\right\rangle+\text { c.c } \\
& =\frac{L_{\mu}}{\Omega} \int_{0}^{1} d \lambda \mathcal{B}(\xi), \quad \xi=\left(\phi_{\mu \nu}, \lambda\right),
\end{aligned}
$$

with $\mathcal{B}(\xi)=i\left(\left\langle\partial_{\lambda} \Phi_{0}^{\lambda} \mid \partial_{\phi_{\mu \nu}} \Phi_{0}^{\lambda}\right\rangle-\left\langle\partial_{\phi_{\mu \nu}} \Phi_{0}^{\lambda} \mid \partial_{\lambda} \Phi_{0}^{\lambda}\right\rangle\right)$. Since $t_{\mu \nu}$ is a bulk property (and we assumed that there is no level crossing for the GS as a function of $\phi_{\mu \nu}$ ), its value should not depend on the boundary conditions when we take the thermodynamic limit. Thus [14]

$$
\left\langle\Delta t_{\mu \nu}\right\rangle=\frac{L_{\mu}}{2 \pi \Omega} \int_{0}^{1} d \lambda \int_{0}^{2 \pi} d \phi_{\mu \nu} \mathcal{B}(\xi)=\frac{L_{\mu}}{2 \pi \Omega} \oint_{\Gamma} \mathcal{A}(\xi) \cdot d \xi
$$

with the line integral performed along the contour $\Gamma$ of $[0,2 \pi] \times[0,1]$ in the plane $\left(\phi_{\mu \nu}, \lambda\right)$, and $\mathcal{A}(\xi)=$ $i\left\langle\Phi_{0}^{\lambda} \mid \nabla_{\xi} \Phi_{0}^{\lambda}\right\rangle$. Assuming a path-independent gauge [1]

$$
\begin{aligned}
\left\langle\Delta t_{\mu \nu}\right\rangle & =\frac{L_{\mu}}{2 \pi \Omega}\left[\gamma_{\mu \nu}^{s}(1)-\gamma_{\mu \nu}^{s}(0)\right] \\
\text { with } \gamma_{\mu \nu}^{s}(\lambda) & =i \int_{0}^{2 \pi} d \phi_{\mu \nu}\left\langle\Phi_{0}^{\lambda} \mid \partial_{\phi_{\mu \nu}} \Phi_{0}^{\lambda}\right\rangle .
\end{aligned}
$$

One can also introduce fluxes along space directions other than $\mu$ and, as long as the gap does not close, average over these additional fluxes. The Berry phases $\gamma_{\mu \nu}^{s}$ are anholonomies associated with the parallel transport of a vector state (GS) in the parameter space determined by the fluxes. Equation (9) shows that the quantum of uncertainty, related to the lack of history in the adiabatic evolution, is at least the inverse of the total transverse section $L_{\mu} / \Omega$. In a periodic system with primitive unit cell volume $\Omega_{0}<\Omega$, the quantum is larger and equal to $L_{\mu}^{0} / \Omega_{0}$, where $L_{\mu}^{0}$ is the length of the unit cell along the $\mu$-direction [11].

The extension to lattice systems is straightforward

$$
\mathbb{H}^{L}=\sum_{\mathbf{r} \mathbf{r}^{\prime}, \alpha \beta} t_{\mathbf{r r}^{\prime}}\left(c_{\mathbf{r} \alpha}^{\dagger}\left[e^{i \theta_{\mu \nu}^{\mathbf{r r}{ }^{\prime}} \sigma^{\nu}}\right]_{\alpha \beta} c_{\mathbf{r}^{\prime} \beta}+\text { H.c. }\right)+\bigoplus_{\mathrm{int}}^{L},
$$

where $c_{\mathbf{r} \alpha}^{\dagger}$ creates an electron of spin $\alpha$ at lattice site $\mathbf{r}$, $\theta_{\mu \nu}^{\mathbf{r r}^{\prime}}=\int_{\mathbf{r}}^{\mathbf{r}^{\prime}} d r_{\mu} A_{\mu \nu}=\phi_{\mu \nu}\left(r_{\mu}^{\prime}-r_{\mu}\right) / L_{\mu}, n_{\mathbf{r}}=\sum_{\alpha} c_{\mathbf{r} \alpha}^{\dagger} c_{\mathbf{r} \alpha}$. The spin current on the bond $\left(\mathbf{r}, \mathbf{r}+\hat{\mathbf{e}}_{\mu}\right)$ is $\mathcal{J}_{\mu \nu}^{s}=$ $\frac{i \hat{\mathbf{e}}_{\mu}}{2} \sum_{\alpha \beta} t_{\mathbf{r r}+\hat{\mathbf{e}}_{\mu}}\left(c_{\mathbf{r}+\hat{\mathbf{e}}_{\mu} \alpha}^{\dagger} c_{\mathbf{r} \beta}-c_{\mathbf{r} \alpha}^{\dagger} c_{\mathbf{r}+\hat{\mathbf{e}}_{\mu} \beta}\right) \sigma_{\alpha \beta}^{\nu}$. The charge Berry phase $\gamma^{c}$ is obtained when $\theta_{\mu \nu}^{\mathbf{r r}^{\prime}} \sigma^{\nu}$ is replaced by $\int_{\mathbf{r}}^{\mathbf{r}^{\prime}} d r_{\mu} A_{\mu}=\phi_{\mu}^{c}\left(r_{\mu}^{\prime}-r_{\mu}\right) / L_{\mu}$, i.e., the flux associated with the usual vector potential $A_{\mu}$ of the electromagnetic field.

Localization Indicators. There is a formal mathematical connection between the geometric phases $\left(\gamma^{c}, \gamma^{s}\right)$ and the localization indicators $\left(z_{L}^{c}, z_{L}^{s}\right)$ [9, 18, 19] that were introduced to discriminate between different phases such 
as conductors and insulators [20]. $z_{L}^{c}$ is defined by Eq. (11) below, replacing $\varphi_{\mu \nu} \boldsymbol{\sigma}^{\nu}$ with a scalar coupling $\varphi_{\mu}^{c}$. For a one dimensional system along the $x$ direction and spin quantization axis $z, z_{L}^{s}$ corresponds to the component $z_{x z}$ of the tensor defined by Eq. (11). The phase of the localization number $z_{L}^{k}$ (with $k=c, s$ ), is related to the Berry phase $\gamma^{k}$ through the relation $\gamma^{k}=\operatorname{Im} \ln z_{L}^{k}$.

Different indicators provide complementary information. They may display different convergence properties to the thermodynamic limit, and most importantly, some indicators are more akin to Monte Carlo methods while others are geared towards numerical renormalization group approaches. The localization parameters $z_{L}^{k}$ and their related Berry phases $\gamma^{k}$ have also been used as sharp topological indicators for establishing quantum phase diagrams of interacting systems [15, 19, 21, 22, 23, 24].

By analogy to $z_{L}^{c, s}$, define the localization indicators

$z_{\mu \nu}\left[\varphi_{\mu \nu}\right]=\left\langle\Psi_{0} \mid \Psi_{0}\left(\varphi_{\mu \nu}\right)\right\rangle,\left|\Psi_{0}\left(\varphi_{\mu \nu}\right)\right\rangle=e^{i \varphi_{\mu \nu} T_{\mu \nu}}\left|\Psi_{0}(\mathbf{0})\right\rangle$,

where $\varphi_{\mu \nu}=2 \pi / L_{\mu}$ and $\left|\Psi_{0}\right\rangle$ is the GS [14].

Considered as a continuous function of $\varphi_{\mu \nu}, z_{\mu \nu}$ plays the role of a characteristic function generating all moments of the $T_{\mu \nu}$ tensor, and trivially satisfies $z_{\mu \nu}[0]=1$, $\left|z_{\mu \nu}\left[\varphi_{\mu \nu}\right]\right| \leq 1, z_{\mu \nu}\left[\varphi_{\mu \nu}\right]=z_{\mu \nu}^{*}\left[-\varphi_{\mu \nu}\right]$. Note, however, that the operator $T_{\mu \nu}$ is not a genuine operator in the Hilbert space bundle defined above, although its exponential is a legitimate one. Therefore, expectation values of arbitrary powers of $T_{\mu \nu}$ have only meaning in terms of $z_{\mu \nu}$ (i.e., there is a quantum of uncertainty [11]). Assuming analyticity in the neighborhood of $\varphi_{\mu \nu}=0$ (i.e., the system has a gap in the thermodynamic limit), $z_{\mu \nu}$ can be written in terms of cumulants $C_{k}\left(T_{\mu \nu}\right)$

$$
z_{\mu \nu}=\exp \left[\sum_{k=1}^{\infty} \frac{\left(i \varphi_{\mu \nu}\right)^{k}}{k !} C_{k}\left(T_{\mu \nu}\right)\right] .
$$

with the end result that

$$
\begin{aligned}
\varphi_{\mu \nu}^{-1} \operatorname{Im} \ln z_{\mu \nu} & =\left\langle T_{\mu \nu}\right\rangle+\mathcal{O}\left(\varphi_{\mu \nu}^{2}\right) \\
-\varphi_{\mu \nu}^{-2} \ln \left|z_{\mu \nu}\right|^{2} & =\left\langle T_{\mu \nu}^{2}\right\rangle-\left\langle T_{\mu \nu}\right\rangle^{2}+\mathcal{O}\left(\varphi_{\mu \nu}^{2}\right) .
\end{aligned}
$$

so that the phase of $z_{\mu \nu}$ is related to the tensor $T_{\mu \nu}$ while its modulus provides information on its quantum fluctuations (it vanishes in the thermodynamic limit for systems with gapless excitations that have a non-zero spin current).

To illustrate an elementary application of Eq. (13), assume that $\left|\Psi_{0}\right\rangle$ is the GS of a periodic system with the primitive unit cell of volume $\Omega_{0}$, (such as that of $\mathrm{LiCoPO}_{4}$ [6] but with spins pointing in the $z$ direction) consisting of two spin up and two down in the direction $z$, and displaced in the direction $x$ so that the contribution to $T_{x z}$ from each unit cell, $\tilde{t}_{x z}=2 \sum_{j=1}^{4} x_{j} S_{j}^{z} \neq 0$ $\left(\left\langle t_{\mu \nu}\right\rangle=\tilde{t}_{\mu \nu} / \Omega_{0}\right)$. Thus, the first member of Eq. (13) should give $N_{u} \tilde{t}_{\mu \nu}$ where $N_{u}$ is the number of unit cells. This equation is invariant if any coordinate $r_{j}^{\mu}$ is replaced by $r_{j}^{\mu}+n L_{\mu}$ with $n$ integer. Equation (11) gives $z_{\mu \nu}=\exp \left(\underset{\sim}{2 \pi i} N_{u} \tilde{t}_{\mu \nu} / L_{\mu}\right)$, and replacing into Eq. (13), $\left\langle T_{\mu \nu}\right\rangle=N_{u} \tilde{t}_{\mu \nu}$ as expected. Note that the same result is obtained if $\tilde{t}_{\mu \nu}$ is replaced by $\tilde{t}_{\mu \nu}+n q$, where $n$ is integer and $q=L_{\mu} / N_{u}$ is the quantum of uncertainty in $\tilde{t}_{\mu \nu}$. However, for translational invariant systems, one can calculate $z_{\mu \nu}$ in the effective one-dimensional problem with fixed total transversal wave vector, $\left(K_{y}, K_{z}\right)$, for which $q=L_{\mu}^{0}$.

Effective single-body schemes. One can implement previous ideas in the framework of spin density (matrix) functional or Hartree-Fock theories. The Hamiltonian acting on the Kohn-Sham (or Hartree-Fock) orbitals, $\psi_{n \mathbf{k}}^{\alpha}(\mathbf{r})$, with lattice periodicity $\boldsymbol{a}$

$$
\sum_{\beta}\left(\frac{\mathbf{p}^{2}}{2 m} \delta_{\alpha \beta}+\hat{v}_{\alpha \beta}(\mathbf{r})\right) \psi_{n \mathbf{k}}^{\beta}(\mathbf{r})=\epsilon_{n \mathbf{k}} \psi_{n \mathbf{k}}^{\alpha}(\mathbf{r})
$$

defines the two-component spinor $\Psi_{n \mathbf{k}}(\mathbf{r})=\left(\begin{array}{c}\psi_{n \mathbf{k}}^{\uparrow}(\mathbf{r}) \\ \psi_{n \mathbf{k}}^{\downarrow}(\mathbf{r})\end{array}\right)$ with the following generalized Bloch-spinor conditions

$$
\begin{aligned}
\boldsymbol{\Psi}_{n \mathbf{k}}\left(\mathbf{r}+a_{\mu} \hat{\mathbf{e}}_{\mu}\right) & =e^{i\left(k_{\mu} a_{\mu}+\frac{\phi_{\mu \nu}}{L_{\mu}} a_{\mu} \sigma^{\nu}\right)} \Psi_{n \mathbf{k}}(\mathbf{r}) \\
\boldsymbol{\Psi}_{n \mathbf{k}}(\mathbf{r}) & =e^{i\left(\mathbf{k}+\frac{\phi_{\mu \nu}}{L_{\mu}} \sigma^{\nu} \hat{\mathbf{e}}_{\mu}\right) \cdot \mathbf{r}} \boldsymbol{u}_{n \mathbf{k}}(\mathbf{r})
\end{aligned}
$$

where $\boldsymbol{u}_{n \mathbf{k}}(\mathbf{r}+\boldsymbol{a})=\boldsymbol{u}_{n \mathbf{k}}(\mathbf{r})$ is the periodic part of the Bloch-spinor. In Eq. (15) $\hat{v}_{\alpha \beta}$ represents the effective one-body potential (which includes the external, Hartree, exchange and correlation components). To simplify the final expression of $\left\langle t_{\mu \nu}\right\rangle$, it is convenient to express the spinor $\boldsymbol{u}_{n \mathbf{k}}$ in the basis of eigenstates of $\sigma^{\nu}$,

$$
\boldsymbol{u}_{n \mathbf{k}}=\left(\begin{array}{c}
u_{n \mathbf{k}}^{\nu+} \\
u_{n \mathbf{k}}^{\nu-}
\end{array}\right), \text { with } \sigma^{\nu} \boldsymbol{u}_{n \mathbf{k}}=\left(\begin{array}{c}
+u_{n \mathbf{k}}^{\nu+} \\
-u_{n \mathbf{k}}^{\nu-}
\end{array}\right),
$$

and the periodic part of the (non-interacting) manyparticle wavefunction along each of the spin directions is the product of Slater determinants: $\Phi_{\mathbf{k}}^{\nu}\left(\mathbf{r}_{1}, \cdots, \mathbf{r}_{N}\right)=$ $\operatorname{Det}\left(A^{\nu+}(\mathbf{k})\right) \operatorname{Det}\left(A^{\nu-}(\mathbf{k})\right)$, where $A_{i j}^{\nu \theta}(\mathbf{k})=u_{i \mathbf{k}}^{\nu \theta}\left(\mathbf{r}_{j}\right)$ with $\theta= \pm, 1 \leq i \leq N_{\theta}$, and $1 \leq j \leq N_{+}\left(1+N_{+} \leq j \leq N\right)$ for $\theta=+(\theta=-)$. $N=N_{+}+N_{-}$is the number of particles in the unit cell. Then, using Eq. (9)

$$
\left\langle t_{\mu \nu}(\lambda)\right\rangle=\frac{i}{(2 \pi)^{3}} \sum_{\theta= \pm} \sum_{n=1}^{\mathrm{N}_{\theta}} \int_{\mathrm{BZ}} d^{3} k \theta\left\langle u_{n \mathbf{k}}^{\nu \theta} \mid \partial_{k_{\mu}} u_{n \mathbf{k}}^{\nu \theta}\right\rangle,
$$

with the momentum integral evaluated over the Brillouin zone (BZ) corresponding to the periodicity $\boldsymbol{a}$. As mentioned above, what has physical meaning is the change in the tensor $t_{\mu \nu}$. Therefore, $\Delta t_{\mu \nu}=t_{\mu \nu}(1)-t_{\mu \nu}(0)$. Here, as in the case of charge and spin macroscopic polarizations [11], the price paid for considering a two-point formula (i.e., forgetting about the $\lambda$-dependent path) is the appearance of a quantum of uncertainty.

Geometry of magnetoelectric response. Finally, to understand the geometric content of the magnetoelectric response, we need to understand the Riemannian structure of our Hilbert space bundle. Consider a set of normalized 
states $\left\{\left|\Psi_{0}(\varphi)\right\rangle\right\}$, where $\varphi$ represents $3 d$ real numbers $\varphi_{\mu \nu}$. Let's assume that this manifold of quantum states is generated by the action of the group of transformations $\left|\Psi_{0}(\boldsymbol{\varphi})\right\rangle=e^{i \boldsymbol{\varphi} \cdot \mathbf{T}}\left|\Psi_{0}(0)\right\rangle$, where $\boldsymbol{\varphi} \cdot \mathbf{T}=\sum_{\mu \nu} \varphi_{\mu \nu} T_{\mu \nu}$. The expectation value of the generalized twist operator $e^{i \boldsymbol{\varphi} \cdot \mathbf{T}}$ measures the character of the spectrum of lowenergy $T_{\mu \nu}$-excitations. Can we find a measure of the distance between two of these quantum states?

Provost and Vallee addressed the problem of establishing a Riemannian structure on an arbitrary differentiable manifold of quantum states [25]. Berry, later on, extended their work by introducing a geometric tensor [26] whose physical relevance in the context of electric charge polarization was addressed in Refs. [18, 27] and also for spin polarization in Ref. [18]. We now introduce a general quantum geometric tensor

$$
G_{\mu \nu ; \alpha \beta}[\varphi]=\left\langle\partial_{\varphi_{\mu \nu}} \Psi_{0}\left|\hat{P}_{0}[\varphi]\right| \partial_{\varphi_{\alpha \beta}} \Psi_{0}\right\rangle
$$

whose real and imaginary parts $G_{\mu \nu ; \alpha \beta}[\varphi]=g_{\mu \nu ; \alpha \beta}[\varphi]+$ $i \Omega_{\mu \nu ; \alpha \beta}[\boldsymbol{\varphi}]$ can be written

$$
\left\{\begin{array}{l}
g_{\mu \nu ; \alpha \beta}[\boldsymbol{\varphi}]=\operatorname{Re}\left\langle\partial_{\varphi_{\mu \nu}} \Psi_{0} \mid \partial_{\varphi_{\alpha \beta}} \Psi_{0}\right\rangle-\gamma_{\mu \nu}[\varphi] \gamma_{\alpha \beta}[\varphi] \\
\Omega_{\mu \nu ; \alpha \beta}[\boldsymbol{\varphi}]=\operatorname{Im}\left\langle\partial_{\varphi_{\mu \nu}} \Psi_{0} \mid \partial_{\varphi_{\alpha \beta}} \Psi_{0}\right\rangle,
\end{array}\right.
$$

with $\hat{P}_{0}[\varphi]=\mathbb{1}-\left|\Psi_{0}(\varphi)\right\rangle\left\langle\Psi_{0}(\varphi)\right|$, and $\gamma_{\mu \nu}[\varphi]=$ $i\left\langle\Psi_{0} \mid \partial_{\varphi_{\mu \nu}} \Psi_{0}\right\rangle$ the Berry connection [1]. The real part of $G_{\mu \nu ; \alpha \beta}[\boldsymbol{\varphi}]$ is a symmetric and positive definite ten- sor representing a generalization of the metric introduced in Ref. [25]. Moreover, it is interesting to remark that the infinitesimal distance is related to the quantum fluctuations of the $T_{\mu \nu}$ tensor, i.e., $g_{\mu \nu, \alpha \beta}(\boldsymbol{\varphi} \rightarrow \mathbf{0})=$ $\left\langle T_{\mu \nu} T_{\alpha \beta}\right\rangle-\left\langle T_{\mu \nu}\right\rangle\left\langle T_{\alpha \beta}\right\rangle$, with expectation values evaluated over $\left|\Psi_{0}(\mathbf{0})\right\rangle \equiv\left|\Psi_{0}\right\rangle$. In a sense, the metric structure on the manifold is fixed by the quantum fluctuations which determine the modulus of $z_{\mu \nu}$ in the thermodynamic limit. On the other hand, the antisymmetric tensor $\Omega_{\mu \nu ; \alpha \beta}[\boldsymbol{\varphi}]=\operatorname{Im}\left\langle\partial_{\varphi_{\mu \nu}} \Psi_{0} \mid \partial_{\varphi_{\alpha \beta}} \Psi_{0}\right\rangle\left(\Omega_{\mu \nu ; \alpha \beta}[\boldsymbol{\varphi}]=\right.$ $\left.-\Omega_{\alpha \beta ; \mu \nu}[\varphi]\right)$ plays the role of a curvature, and is a quantity connected to the non-dissipative part of the spin conductance in adiabatic transport.

In summary, we have introduced a formalism that not only leads to a geometric understanding of a spin component of the magnetoelectric response but also provides a computational method to study changes in ferrotoroidic moment, $\Delta \boldsymbol{\tau}$, when the system under consideration has periodic boundary conditions (no surface). Forgetting about the history of the evolution leading to $\Delta \boldsymbol{\tau}$ amounts to the appearance of a quantum of uncertainty of magnitude $\frac{\mu_{B}}{4} \frac{L_{\mu}^{0}}{\Omega_{0}}$. Our many-body formalism generalizes, giving an operational physical interpretation to, the concept of spin Berry phase introduced in [13], and should be particularly useful within the framework of spin density (matrix) functional or Hartree-Fock theories.

LANL is supported by US DOE under Contract No. W-7405-ENG-36.
[1] L. D. Landau, E. M. Lifshitz, and L. P. Pitaevskii, Electrodynamics of Continuous Media, Vol. 8 (ButterworthHeinemann, Oxford, 1996).

[2] H. Schmid, Ferroelectrics 252, 41 (2001); H. Schmid, in Magnetoelectric Interaction Phenomena in Crystals, edited by M. Fiebig, V. Eremenko, and I. E. Chupis (Kluwer, Dordrecht, 2004), pp. 1-34.

[3] M. Fiebig, J. Phys. D 38, R123 (2005).

[4] T. Arima et al., J. Phys. Soc. Jpn. 74, 1419 (2005).

[5] K. Sawada and N. Nagaosa, Phys. Rev. Lett. 95, 237402 (2005).

[6] B. B. Van Aken et al., Nature (London) 449, 702 (2007).

[7] C. Ederer and N. A. Spaldin, Phys. Rev. B 76, 214404 (2007).

[8] V. M. Dubovik and V. V. Tugushev, Phys. Rep. 187, 145 (1990).

[9] A. A. Aligia and G. Ortiz, Phys. Rev. Lett. 82, 2560 (1999).

[10] H. Schmid, Ferroelectrics 252, 41 (2001).

[11] G. Ortiz and R. M. Martin, Phys. Rev. B 49, 14202 (1994).

[12] In the context of non-interacting (single-particle) theories it was originally introduced by R. D. King-Smith and D. Vanderbilt, Phys. Rev. B 47, 1651 (1993), and R. Resta, Europhys. Lett. 22, 133 (1993).

[13] A. A. Aligia, Europhys. Lett. 45, 411 (1999).

[14] Since we are interested in changes of the ferrotoroidic spin moment, the formalism can be extended to evolutions with constant non-zero magnetization per unit cell $m_{0}=$ $2 \int_{\Omega_{0}} d^{3} r \mathcal{S}_{\nu}(\mathbf{r})$. If $m_{0}=\mathrm{m} / \ell$, is an irreducible fraction ( $\mathrm{m}, \ell$ are integers), symmetry arguments [9, 13] show that the integrals in $\phi_{\mu \nu}$ should be extended to $2 \pi \ell$ and $\varphi_{\mu \nu}=$ $2 \pi \ell / L_{\mu}$ in $z_{\mu \nu}$ [Eq. (11)].

[15] G. Ortiz et al., Phys. Rev. B 54, 13515 (1996).

[16] D. J. Thouless, Phys. Rev. B 27, 6083 (1983).

[17] From the local relation $\sum_{\mu} \partial_{\mu} \mathcal{T}_{\mu \nu}(\mathbf{r})=-2 \mathcal{S}_{\nu}(\mathbf{r})$ and the continuity equation, it becomes clear that the spin current $\mathcal{J}_{\mu \nu}^{s}(\mathbf{r})$ is defined up to the addition of a solenoidal field $\nabla \times \mathbf{f}(\mathbf{r})$ where the vector field $\mathbf{f}(\mathbf{r})$ satisfies the gauge condition $\nabla \cdot \mathbf{f}(\mathbf{r})=0$. This additional term represents zero divergence spin currents (closed loops), such as the edge currents existing in certain insulators, and does not contribute to Eq. (6) 11].

[18] G. Ortiz and A. A. Aligia, Phys. Stat. Solidi 220, 737 (2000).

[19] A. A. Aligia et al., J. Low Temp. Phys. 117, 1747 (1999); ibid Phys. Rev. B 61, 7883 (2000).

[20] R. Resta ans S. Sorella, Phys. Rev. Lett. 82, 370 (1999).

[21] A. A. Aligia et al., Phys. Rev. Lett. 99, 206401 (2007).

[22] C. D. Batista and A. A. Aligia, Phys. Rev. Lett. 92, 246405 (2004); ibid, Phys. Rev. B 71, 125110 (2005).

[23] M. E. Torio et al., Phys. Rev. B 73, 115109 (2006).

[24] M. Nakamura and S. Todo, Phys. Rev. Lett. 89, 077204 (2002).

[25] J.P. Provost and G. Vallee, Commun. Math. Phys. 76, 289 (1980). 
[26] M. V. Berry, in Geometric Phases in Physics, edited by A. Shapere and F. Wilczek (World Scientific, Singapore, 1989).
[27] I. Souza, T. Wilkens, and R. M. Martin, Phys. Rev. B 62, 1666 (2000). 